\title{
Improved Transfection Efficiency and Metabolic Activity in Human Embryonic Stem Cell Using Non-Enzymatic Method
}

\author{
C-Yoon Kim ${ }^{1,3, *}$, In-Kyu Hwang ${ }^{1, *}$, Changhee Kang ${ }^{1}$, Eun-Bin Chung, \\ Cho-Rok Jung ${ }^{2}$, Hanseul $\mathrm{Oh}^{3}$, Young-Hoon Jeong ${ }^{1}$, Sung-Hwan Moon ${ }^{1}$, \\ Jong Soo Kim ${ }^{1}$, Ki-Sung Hong ${ }^{1}$, Jae-Hak Park ${ }^{3}$, Hyung-Min Chung ${ }^{1}$ \\ ${ }^{1}$ Department of Stem Cell Biology, School of Medicine, Konkuk University, Seoul, Korea \\ ${ }^{2}$ Gene Therapy Research Unit, Korea Research Institute of Bioscience and Biotechnology, Daejeon, Korea \\ ${ }^{3}$ Department of Laboratory Animal Medicine, College of Veterinary Medicine, Seoul National University, Seoul, Korea
}

Human embryonic stem cells (hESCs) are pluripotent cells widely used in conventional and regenerative medicine due to their ability to self-renew, proliferate and differentiate. Recently, genetic modification of stem cells using genome editing is the most advanced technique for treating hereditary diseases. Nevertheless, the low transfection efficiency of hESCs using enzymatic methods is still limited in in vitro preclinical research. To overcome these limitations, we have developed transfection methods using non-enzymatic treatments on hESCs. In this study, hESCs were transfected following enzymatic (TrypLE and trypsin) and non-enzymatic treatment ethylenediaminetetraacetic acid (EDTA) to increase transfection efficiency. Flow cytometric analysis using an enhanced green fluorescent protein vector showed a significantly increased transfection efficiency of EDTA method compared to standard enzyme method. In addition, the EDTA approach maintained stable cell viability and recovery rate of hESCs after transfection. Also, metabolic activity by using Extracellular Flux Analyzer revealed that EDTA method maintained as similar levels of cell functionality as normal group comparing with enzymatic groups. These results suggest that transfection using EDTA is a more efficient and safe substitute for transfection than the use of standard enzymatic methods.

Keywords: Human embryonic stem cell, Transfection, EDTA, Metabolic activity

\section{Introduction}

Human embryonic stem cells (hESCs) are isolated from

Received April 24, 2018, Revised July 12, 2018,

Accepted July 12, 2018, Published online August 31, 2018

Correspondence to Hyung-Min Chung

Department of Stem Cell Biology, School of Medicine, Konkuk University, 120 Neungdong-ro, Gwangiin-gu, Seoul 05029, Korea Tel: +82-2-2049-6257, Fax: +82-2-455-9012

E-mail: hmchung@kku.ac.kr

${ }^{*}$ These authors contributed equally to this work.

(a) This is an open-access article distributed under the terms of the Creative Commons Attribution Non-Commercial License (http://creativecommons.org/ licenses/by-nc/4.0/), which permits unrestricted non-commercial use, distribution, and reproduction in any medium, provided the original work is properly cited.

Copyright (C) 2018 by the Korean Society for Stem Cell Research the inner cell mass of human blastocysts and exhibit unlimited self-renewal in addition to several cell differentiation characteristics $(1,2)$. hESCs with these characterizations are used in basic and clinical medicine studies, especially in vivo/in vitro cell transplantation, toxicity testing or drug screening, as well as establishment of model systems of human disease. Moreover, genetic modification of hESC has allowed for the control of differentiation or other molecular mechanisms (3-5).

Recent advances in stem cell research with genetic manipulation provide crucial information regarding stagespecific gene expression during differentiation (6-9). Thus, several genetic modification methods such as chemical-based transfection, electroporation, and especially use of viral vectors have been developed (10-13). However, although viral transfection is a highly efficient method, 
there is a critical risk of mutation or teratoma formation after inserting the viral gene (14). Therefore, chemical transfection reagents and electroporation methods have been developed and used for hESCs research.

Unfortunately, transfection efficiency of hESC is still much lower than that of somatic cells because of its characteristic of growing compact colonies. Various methods (e.g. trypsin adaptation (10) and DMSO treatment (15) have been suggested to improve hESC transfection efficiency for transgenic applications, but these methods carry a risk of causing karyotypic abnormality and decreased cell viability (16-18). Therefore, there is a need to develop alternative technologies to successfully improve the transfection efficiency of hESCs.

Ethylenediaminetetraacetic acid (EDTA) is a chelating agent that binds metallic ions and loosens the cell-cell junctions to increase the contact surface. Recently, it has been reported that hESC can be successfully passaged using non-enzymatic reagent EDTA with better cell viability compared with other methods, without risk of karyotypic abnormality (19). With this background, we proposed that EDTA method has potential for application in genetic modification of hESCs.

In this study, we evaluated the EDTA as a new transfection method and confirmed both improved transfection efficiency and stable cell viability on hESCs. These results demonstrate that usage of EDTA is safe substitute for transfection than that of enzymatic methods.

\section{Materials and Methods}

\section{hES cell culture in feeder-free condition}

H9 hESC (Wicell, WI) was cultured on vitronectincoated $100 \Phi$ plates in essential 8 media (Thermo Fisher Scientific) at $37^{\circ} \mathrm{C}$ in $5 \% \mathrm{CO}_{2}$. To adapt on feeder-free condition, hESCs on mouse embryonic fibroblasts were transferred to vitronectin-coated plates and passaged $5 \sim 6$ times to remove mouse embryonic fibroblasts. hESCs were detached by using EDTA $(0.1 \mathrm{mM})$ in phosphate buffered saline (PBS) without $\mathrm{Ca}^{2+} / \mathrm{Mg}^{2+}$ and transferred new culture plates. Passaging of hESC is performed twice per week and media change was changed daily.

\section{Immunocytochemistry of hESC}

hESCs were fixed with 4\% paraformaldehyde for $20 \mathrm{mi}$ nutes and then permeabilized with $0.1 \%$ Triton $\mathrm{X}-100$ in PBS for $5 \mathrm{~min}$. After a treatment with $5 \%$ normal goat serum for $30 \mathrm{~min}$, the cells were incubated with primary antibodies against one of the pluripotency markers mouse anti-Oct-4 (Santa Cruz Biotechnology Inc., CA, 1:250) and rabbit anti-Nanog (Santa Cruz Biotechnology Inc, 1:250), for $18 \mathrm{~h}$ at $4^{\circ} \mathrm{C}$. The cells were then washed 3 times with PBS and incubated with Alexa Fluor 488- conjugated secondary antibodies (Invitrogen, NY, 1:1000) for $1 \mathrm{~h}$. After washing 3 times with PBS, DAPI (Invitrogen) staining was performed for 5 minutes. The samples were immediately imaged using epi-fluorescence microscopes (Nikon TE 2000-U, Nikon, Japan).

\section{Karyotyping}

In order to analyze the hESC chromosome normality, H9 hESC was cultured from passage 54 and H9 hESC (passage 68) was karyotyped by Gendix Inc in Seoul, Korea. hESCs confluence in $100 \Phi$ vitronectin-coated dish was $\sim 90 \%$ to analyze chromosome normality.

\section{Lipofection assay}

Lipofectamine 2000 was used following instructions. GFP-expressing vector was used to pmaxGFP containing 4D-nucleofector kit (Lonza; Walkersville, MD). The DNA complex was consisted of $2 \mu \mathrm{g}$ plasmid DNA and $100 \mu \mathrm{l}$ Opti-MEM (Invitrogen) per well. And after that, DNA complex was added to lipofectamine complex at a 1:1 ratio and DNA-lipid complex was incubated for 5 minutes at RT. hESCs on vitronectin-coated plates were pre-treated for 5 minutes prior to lipofection during DNA-lipid complex incubation in RT. Each well was washed by PBS and $10 \mathrm{mM}$ EDTA in PBS without $\mathrm{Ca}^{2+} / \mathrm{Mg}^{2+}, 0.5 \times$ TrypLE and $0.025 \%$ trypsin (Invitrogen) were treated each well for 5 minutes. And dissociation reagents were replaced with essential 8 media gently. $250 \mu 1$ of DNA-lipid complex was added per well and incubated for 24 hours at $37^{\circ} \mathrm{C}$ in $5 \% \mathrm{CO}_{2}$. Then transfected cells were analyzed by using fluorescence microscope and fluorescence activated cell sorting (FACS) analysis to quantify the transfected cell amounts.

\section{Electroporation assay}

hES cells were treated with the ROCK inhibitor Y-27632 $1 \mathrm{~h}$ and harvested using TrypLE. We used the P3 Primary Cell 4D-Nucleofector ${ }^{\circledR}$ X-Kit L (Lonza) and applied 4D-nucleofector device (Lonza). We transfected $8 \times 10^{5}$ cells $/ \mathrm{ml}$ with $2 \mu \mathrm{g}$ of pmaxGFP vector (Lonza). Harvested cells were re-suspended using $100 \mu 1$ of nucleofector solution containing pmaxGFP vector and $100 \mu 1$ of mastermix was carried into cuvette. After electroporation, $1 \mathrm{ml}$ of pre-warmed media was added into cuvette and mixed by gently pipetting up and down $2 \sim 3$ times before incubation $\left(24\right.$ hours at $37^{\circ} \mathrm{C}$ in $5 \% \mathrm{CO}_{2}$ ). Depending on cell density, re-suspended cells were seeded onto vi- 
tronectin-coated plates and analyzed at least $4 \sim 8$ hours later. Transfected cell amounts were also quantified using FACS analysis.

\section{Flow cytometry analysis}

Transfected cells were treated with the ROCK inhibitor Y-27632 (10 mM) for 1 hour to increase cell survival before dissociation. Cells were washed by PBS and by using TrypLE for $3 \sim 5$ minutes at $37^{\circ} \mathrm{C}$ in $5 \% \mathrm{CO}_{2}$, cells were dissociated to create a single cell suspension state. Cells were re-suspended in $\mathrm{Ca}^{2+} / \mathrm{Mg}^{2+}$-free PBS containing 5\% fetal bovine serum (Invitrogen). Re-suspended single cells were analyzed on a FACSCalibur system (BD Biosciences; San Jose, CA) by applying BD CellQuest software.

\section{Cell viability \& recovery rate}

Cell viability measurement using $0.4 \%$ trypan blue solution (Gibco BRL, MD) was performed by standard solution. Trypan blue solution was mixed with 1:1 ratio, using $10 \mu 1$ of stain to $10 \mu 1$ of cell suspension. Mixed sample was loaded into a Neubauer hemocytometer and counted with a light microscope (Nikon). And after transfection, cell counting was performed to make single cell by TrypLE. And respective cells were calculated the percentage of viability/recovery.

\section{Glycolytic extracellular acidification rate analysis}

Glycolysis and glycolytic capacity were determined for PC3M and PCS cells using the Seahorse Extracellular Flux (XF-96) analyzer (Seahorse Bioscience, Chicopee, MA). Cells were cultured for 2 hours in the absence of glucose. Three sequential injections of D-glucose $(2 \mathrm{~g} / \mathrm{L})$, oligomycin $(1 \mu \mathrm{M})$, and 2-Deoxyglucose $(100 \mathrm{mM})$ provided extracellular acdification (ECAR) associated with glycolysis, the maxiumum glycolytic capacity, and nonglycolytic ECAR. Glycolysis was defined as ECAR following the addition of D-glucose and maximum glycolytic capacity was defined as ECAR following the addition oligomycin. ECAR following treatment with 2-Deoxyglucose is associated with non-glycolytic activity.

\section{Real-time polymerase chain reaction}

Total RNA was extracted using TRI reagent (Sigma) according to the manufacturer's instructions. First-strand cDNA was synthesized from $1 \mu \mathrm{g}$ of RNA using the iScript cDNA Synthesis kit (Bio-Rad, Hercules, CA). cDNA from various cell samples were amplified by real-time quantitative PCR (Q-PCR) with specific primers (PKM2: 5'-TCGGAGGTTTGATGAAAT-3' and 5'-TCTCC AGCATCTGA GTAG-3'; LDHA: 5'-GGTTGAGAGTGCT
TATGA-3' and 5'-AACACTAAGGAAGACATCA-3'; GLU T1: 5'-AGTATGTGGAGCAACTGTGT-3' and 5'-TTGAA GTAGGTGAAGATGAAGAAC-3'; HK1: 5'-GGTGAAATC GTCCGCAAC-3' and 5'-CCCGGGTCTTCATCGTC-3') and the data was normalized GAPDH (5'-CCTCAACTAC ATGGTTTACA-3' and 5'-CTCCTGGAAGATGGTGAT-3').

\section{Statistical analysis}

One-way analysis of variance (ANOVA) followed by the Tukey HSD test was performed in SPSS Statistics (version 16). Experimental data were checked to determine if there was a significant difference. The level for statistical significance was set at the 0.05 and 0.01 . All data are shown as mean \pm standard error.

\section{Results}

\section{Karyotyping analysis during hESC passaging and characterization of hESCs}

Immunocytochemistry assay was performed in order to identify pluripotency of $\mathrm{H} 9$ cell line that passaged by EDTA. Consequently, H9 hESCs were expressed Oct4 and Nanog grew on a feeder layer (Supplemental Fig. 1). And, by using this characterized cell line, H9 feeder-free hESCs (passage 68) that passaged more than 10 times by EDTA had a normal karyotype (Supplemental Fig. 2).

\section{Comparison of transfection methods to improve transfection efficiency using enzymatic and non-enzymatic reagents}

Feeder-free hESCs were passaged at least 5 to 6 times to remove the Mouse embryonic fibroblasts (MEFs). In order to improve hESC transfection efficiency, we developed a method to loosen cell-cell junctions of hESCs using trypsin, TrypLE and EDTA.

After pre-treatment with Lipofectamine 2000 for $5 \mathrm{mi}$ nutes, transfection groups were divided into normal (untreated), trypsin, TrypLE, and EDTA groups. Lipofection was performed for 24 hours using the DNA-lipid complex and transfection efficiencies of respective groups were compared. We confirmed fluorescein isothiocyanate (FITC) expression by transfected hESCs in each group using a fluorescence microscope. The quantity of transfected cells showing florescence was quantified using fluorescence-activated cell sorting (FACS) analysis. As shown in Fig. 1B, transfection efficiencies were calculated respectively (normal: $6.17 \pm 1.4 \%$, trypsin: $15.21 \pm 1.6 \%$, TrypLE: $18.95 \pm 0.67 \%$, and EDTA: $23.81 \pm 2.83 \%$ ). All pre-treated groups showed higher transfection efficiency compared with the normal group. Of note, the transfection 

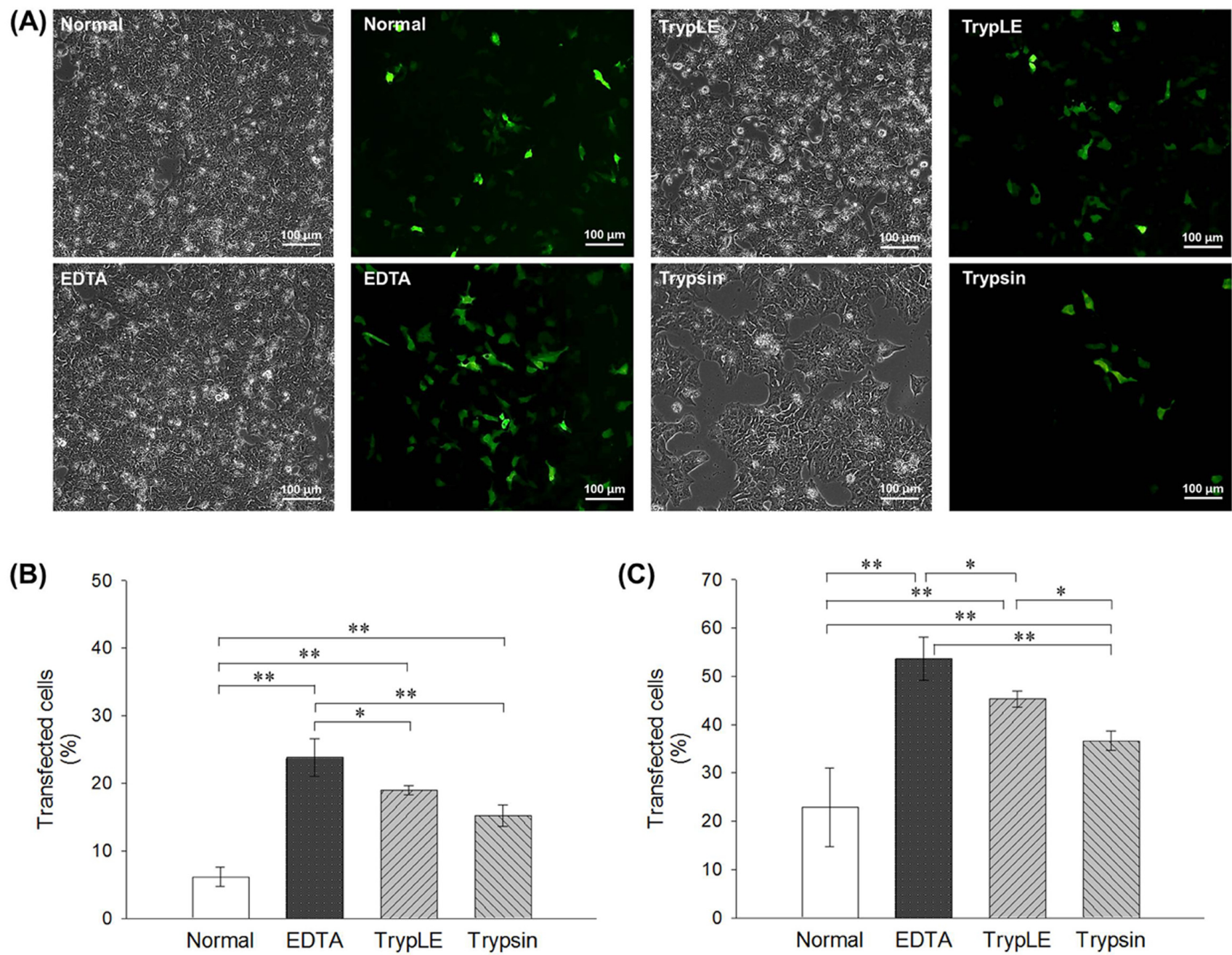

Fig. 1. Comparison of electroporation groups of hESCs (H9) using GFP-fluorescence (green) microscope (A). FACS analysis data of lipofection (B) and electroporation (C). Scale bar: $100 \mu \mathrm{m}$; Data represent the mean \pm SD. ${ }^{*} p<0.05,{ }^{*} \mathrm{p}<0.01$.

efficiency of EDTA group was very similar to that of TrypLE group.

Cell detachment was performed using trypsin, TrypLE, and EDTA groups to perform electroporation, whereas in the normal group the cells were mechanically detached using a glass pipette. Transfection efficiency was confirmed at 24 hours after transfection by identifying the FITC-expressing transfected cells (Fig. 1A) and by calculating expression rates using FACS, as shown in Fig. 1C (normal: $22.91 \pm 8.17 \%$, trypsin: $36.6 \pm 1.98 \%$, TrypLE: $45.34 \pm 1.69 \%$, and EDTA: $53.71 \pm 4.46 \%$ ). Similar to the results obtained with lipofection, all treated groups showed higher transfection efficiency, with similar values obtained for EDTA and TrypLE groups.

\section{Non-enzymatic reagent increases cell viability and recovery compared with enzymatic reagents}

Once cells are transfected, cell damage is inevitable and most transfected cells are hard to attach compared with normal group. Cell viability is essential for cell maintenance after transfection. Therefore, we analyzed cell survival rate and cell recovery in each group after transfection.

To assess hESC recovery after transfection, hESCs in each group were quantified using a low-magnification microscopic image $(\times 40)$ after 24 hours of re-attachment (Fig. 2A). The trypsin group showed the lowest cell recovery, while EDTA group showed a similar rate as the normal group. Additionally, cell survival rates after transfection were calculated using cell counting kit-8. As shown in Fig. 2B, trypsin and TrypLE groups showed low surviv- 

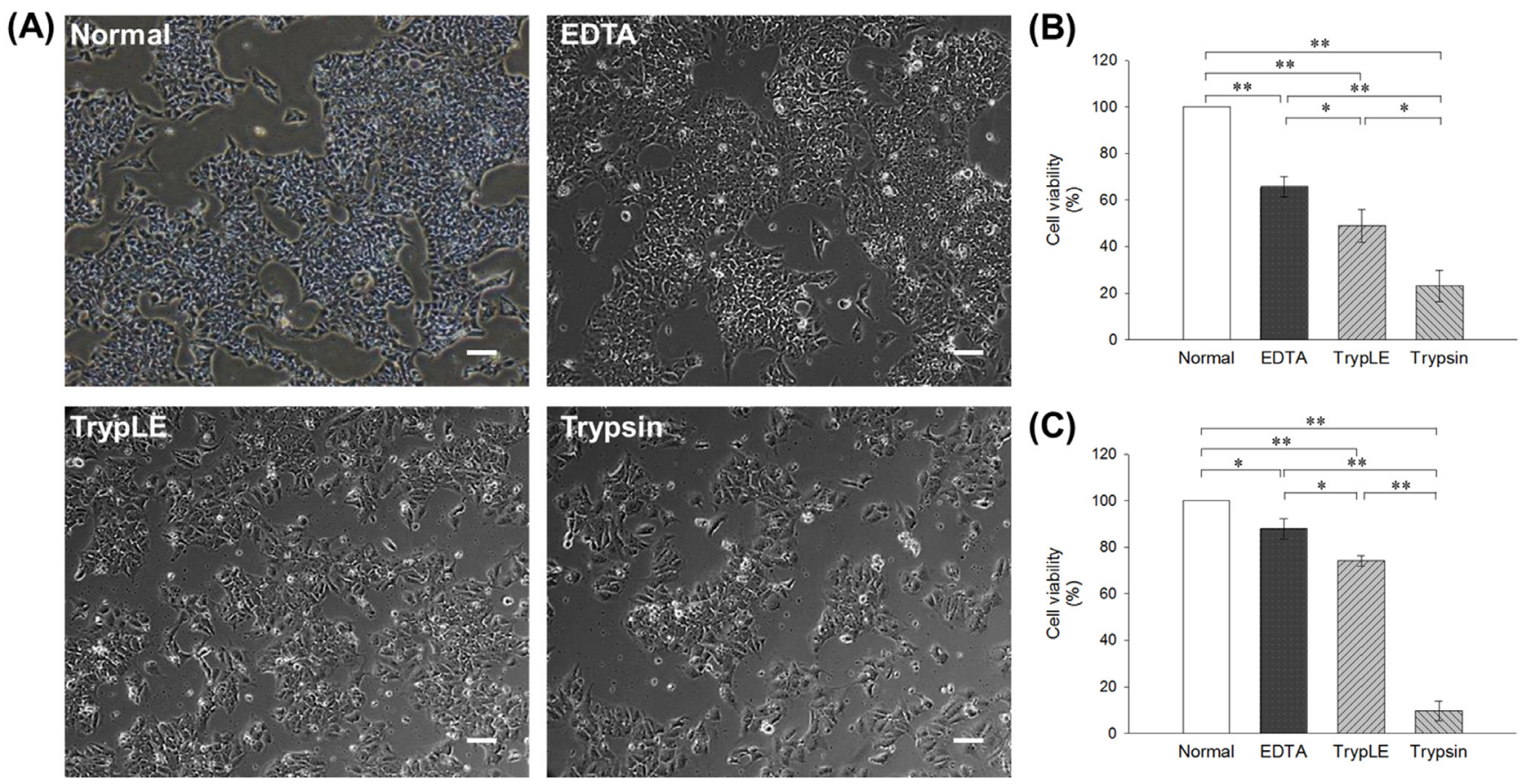

Fig. 2. Bright field image indicating cell re-attachment after transfection (A). Lipofection transfected cell viability (B) and electroporation transfected cell viability (C). Data were normalized to normal control as $100 \%$. Scale bar: $50 \mu \mathrm{m}$; Data represent the mean \pm SD. ${ }^{*} \mathrm{p}<0.05$, $* * \mathrm{p}<0.01$.

al rate (trypsin: $13.36 \%$ and TrypLE: $38.46 \%$ ) compared with normal group; however, EDTA (64.78\%) group exhibited increased survival rate after lipofection transfection. In addition, EDTA group (89.9\%) showed a similar cell survival rate after electroporation to that of normal group as compared to other enzymatic groups (trypsin: 3.97\% and TrypLE: 68.85\%), as shown in Fig. 2C. These results imply that EDTA method causes less damage to hESCs and efficient re-attachment than other enzymatic methods after transfection.

\section{Glycolytic extracellular acidification rate analysis}

In order to determine the metabolic functionality of in hESCs in each group, glycolytic extracellular acidification (ECAR) was also analyzed by Extracellular Flux Analyzer (Fig. 3A). In the presence of glucose, glycolytic acidification of the extracellular space in EDTA group occurred at similar rate to normal group as compared to the other groups (Fig. 3B). In addition, glycolytic capacity, which is the ability of the glycolytic pathway to upregulate in time of energy need, was assessed after the addition of oligomycin that is a specific inhibitor of the mitochondrial ATP synthase. Glycolytic capacity was also similar in EDTA group to normal group as compared with the other groups (Fig. 3B). The addition of 2-deoxyglucose (2-DG), an inhibitor of glycolysis, was used to confirm that the
ECAR measured was a result of glycolytic metabolism. ECAR was restored to non-glycolytic levels in both cell lines following 2-DG treatment. Collectively, these results indicate that EDTA has the least stress on metabolic function, compared to other enzymatic treatments.

\section{Discussion}

Undifferentiated hESCs and hESC-derived differentiated cells can be used as cell therapy in conventional and regenerative medicine studies. Hence, transgenic methods have been developed to induce stable expression after specific gene insertion. Several gene transfection methods have been reported, using lipofection, electroporation, or viral vectors to insert specific genes into target cells. Among these, lipofection has the advantage of maintaining cell viability and functionality, however, the transfection efficiency of hESCs is inadequate. Electroporation method shows high transfection efficiency, but cell survival rate is low due to cell damage during transfection $(10,16,20)$. As an alternative, to reduce cell damage and improve transfection efficiency, several alternatives such as treatment with DMSO, culture condition modifications, and amendments to transfection protocol have been suggested $(10,15)$, but these methods have proved inadequate to improve transfection efficiency. 
(A)

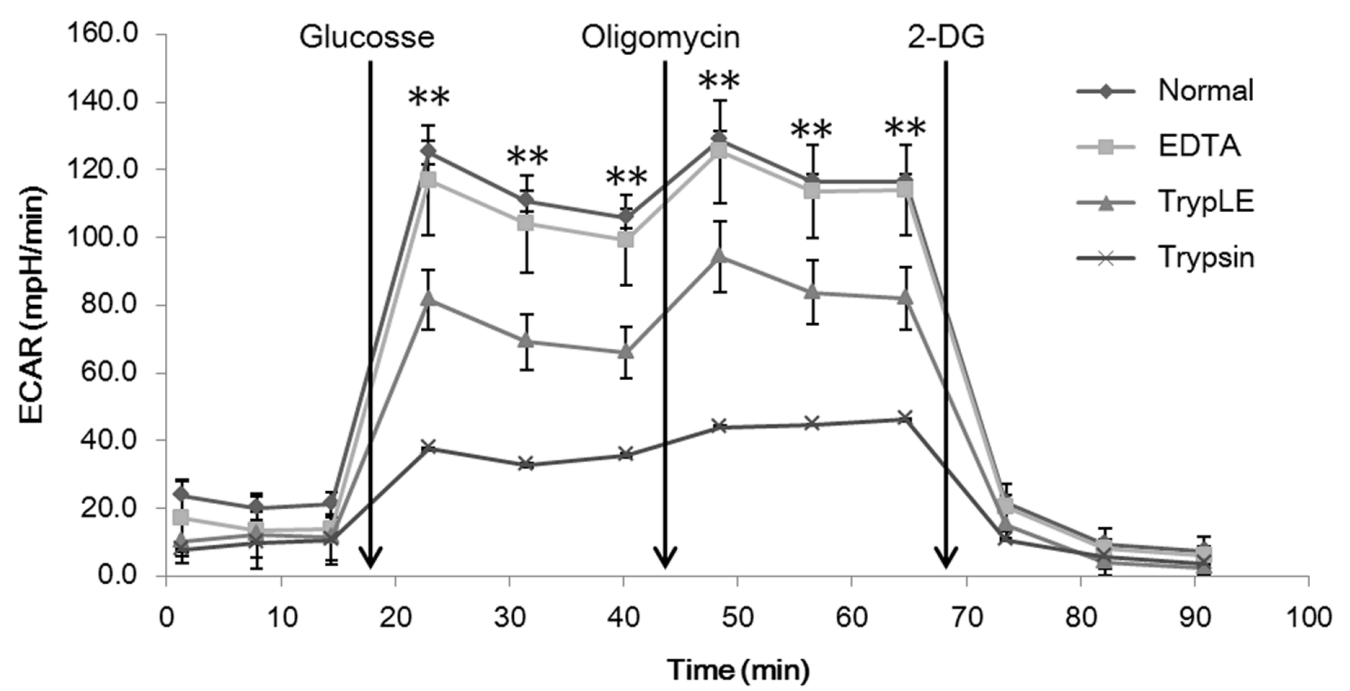

(B)
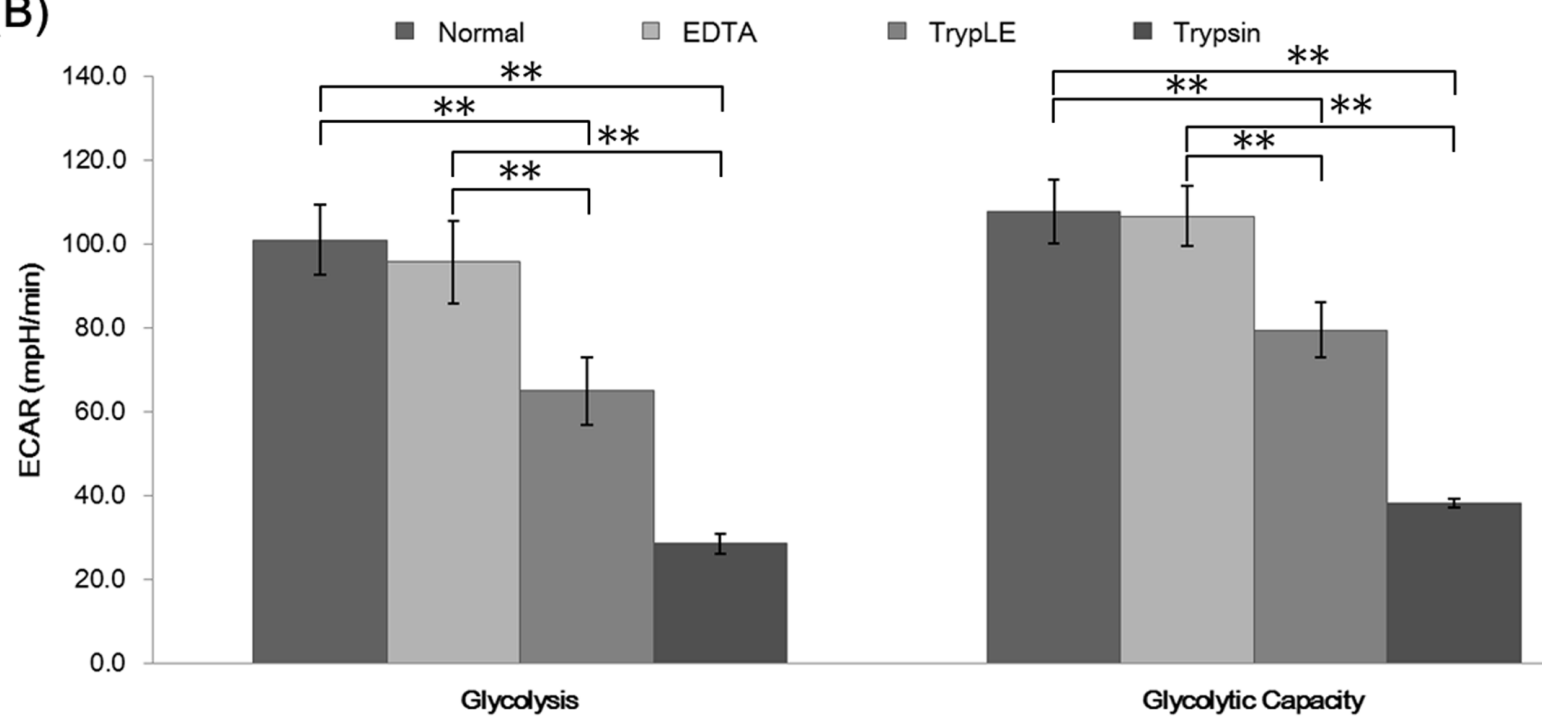

Fig. 3. Extracellular acidification rate (ECAR) comparing with lipofection groups (Normal, EDTA, TrypLE and trypsin) (A). Data between EDTA and TrypLE groups represent the mean \pm SD. ${ }^{* *} p<0.01$. Extracellular acidification rate (ECAR) comparing with lipofection groups (EDTA, TrypLE and trypsin) (B). Mean glycolytic ECAR and glycolytic capacity \pm SD. ${ }^{*} p<0.05,{ }^{* *} p<0.01$.

EDTA plays a role in loosening cell-to-cell junctions in hESCs during passaging. Compared with the commonly used, enzymatic, TrypLE method, EDTA treatment has an advantage of causing lesser cell damage, showing a high cell survival rate during passaging as well as normal karyotype even after long-term cell culture (Supplemental Fig. 2). In addition, EDTA passaged hESCs-derived EBs had a pluripotency (Supplemental Fig. 1). Based on this background, we evaluated the feasibility of EDTA treatment to improve gene transfection efficiency in hESCs.

After lipofection using lipofectamine 2000, we observed improved transfection efficiency (Fig. 1B). Since dis- sociation factors such as EDTA act via loosening the tight junctions between hESC cell surfaces, we proposed that treatment with a dissociation factor would improve hESC transfection efficiency. Electroporation was conducted in addition to treatment with trypsin, EDTA, and TrypLE, which are agents generally used for cell detachment. Electroporation showed no significant difference in transfection efficiencies between normal and EDTA groups (Fig. 1C). These results imply that enzymatic reagents can be substituted by non-enzymatic ones during transfection.

Nevertheless, chemical reagents such as trypsin and TrypLE not only play a role in loosening cell adhesions 
but also affect hES cell conditions, especially cell metabolism (21). Therefore, to evaluate the hES cell condition after treatment with dissociation reagents, cell viability and recovery were confirmed in each treated group. As shown in Fig. 2B and C, cell survival rates of trypsin and TrypLE groups were significantly decreased compared with other groups. Moreover, cell recovery after 24 hours of transfection indirectly showed hESC damage caused by trypsin and TrypLE. In contrast, EDTA group showed stable cell viability and steady cell re-attachment after transfection (Fig. 2B and C). These results are consistent with previous reports regarding beneficial effects of EDTA on hESC passaging, where EDTA functions as a calcium chelator, removing $\mathrm{Ca}^{2+}$ ions and thereby loosening intercellular junctions, which consequently increases the cell surface area without causing damage to the cell. In addition, glycolytic extracellular acidification rate was analyzed to evaluate the mitochondrial functionality of transfected hESCs. ECAR results showed that the EDTA group had similar levels to that of the normal group in terms of glycolytic function and EDTA, also, showed that EDTA group had higher levels than any other 2 groups on each injection stage (Fig. 3). The mRNA levels of LDHA, PKM2, GLUT1 and HK1 also supported the increased mitochondrial function in EDTA group (Supplemental Fig. 3). With this background, we proposed that EDTA could serve as an efficient and stable transfection tool for hESCs.

Though TrypLE is commonly used for transfection and cell passaging, the risk of genetic abnormalities as well as cell death or damage to cell functionality is a matter of concern, rendering the method unsuitable for regenerative medicine studies. So, we demonstrated higher cell viability and cell recovery rates using EDTA. EDTA is safe and easy to use for cell maintenance and genetic modification as compared to enzymatic reagents. Furthermore, EDTA affects less damage than other dissociation factors to the mitochondrial respiratory functionality in cell. The results of this study demonstrate that EDTA is not only a suitable substitute but also overcomes the drawbacks of enzymatic reagents. Thus, EDTA treatment has potential applications in the development of cell therapy products and regenerative medicine studies.

\section{Acknowledgments}

This research was supported by the Bio \& Medical Technology, Development Program of the NRF funded by the Korean government MSIP (2015M3A9C7030091) and the KRIBB Research Initiative Program and the Research Center for Production Management and Technical Devel- opment for High Quality Livestock Products through Agriculture, Food and Rural Affairs Research Center Support Program (715003-07), Ministry of Agriculture, Food and Rural Affairs.

\section{Potential Conflict of Interest}

The authors have no conflicting financial interest.

\section{Supplementary Materials}

Supplementary data including three figure can be found with this article online at http://pdf.medrang.co.kr/paper/ pdf/IJSC/IJSC-11-s18037.pdf.

\section{References}

1. Thomson JA, Itskovitz-Eldor J, Shapiro SS, Waknitz MA, Swiergiel JJ, Marshall VS, Jones JM. Embryonic stem cell lines derived from human blastocysts. Science 1998;282: 1145-1147

2. Reubinoff BE, Pera MF, Fong CY, Trounson A, Bongso A. Embryonic stem cell lines from human blastocysts: somatic differentiation in vitro. Nat Biotechnol 2000;18:399-404

3. Braam SR, Denning C, Matsa E, Young LE, Passier R, Mummery CL. Feeder-free culture of human embryonic stem cells in conditioned medium for efficient genetic modification. Nat Protoc 2008;3:1435-1443

4. Costa M, Dottori M, Sourris K, Jamshidi P, Hatzistavrou T, Davis R, Azzola L, Jackson S, Lim SM, Pera M, Elefanty AG, Stanley EG. A method for genetic modification of human embryonic stem cells using electroporation. Nat Protoc 2007;2:792-796

5. Giudice A, Trounson A. Genetic modification of human embryonic stem cells for derivation of target cells. Cell Stem Cell 2008;2:422-433

6. Pons J, Huang Y, Takagawa J, Arakawa-Hoyt J, Ye J, Grossman W, Kan YW, Su H. Combining angiogenic gene and stem cell therapies for myocardial infarction. J Gene Med 2009;11:743-753

7. Rufaihah AJ, Haider HK, Heng BC, Ye L, Tan RS, Toh WS, Tian XF, Sim EK, Cao T. Therapeutic angiogenesis by transplantation of human embryonic stem cell-derived CD133+ endothelial progenitor cells for cardiac repair. Regen Med 2010;5:231-244

8. Somers A, Jean JC, Sommer CA, Omari A, Ford CC, Mills JA, Ying L, Sommer AG, Jean JM, Smith BW, Lafyatis R, Demierre MF, Weiss DJ, French DL, Gadue P, Murphy GJ, Mostoslavsky G, Kotton DN. Generation of transgene-free lung disease-specific human induced pluripotent stem cells using a single excisable lentiviral stem cell cassette. Stem Cells 2010;28:1728-1740

9. Zwaka TP, Thomson JA. Homologous recombination in human embryonic stem cells. Nat Biotechnol 2003;21:319-321

10. Braam SR, Denning C, van den Brink S, Kats P, 
Hochstenbach R, Passier R, Mummery CL. Improved genetic manipulation of human embryonic stem cells. Nat Methods 2008;5:389-392

11. Eiges $R$, Schuldiner $M$, Drukker $M$, Yanuka $O$, Itskovitz-Eldor J, Benvenisty N. Establishment of human embryonic stem cell-transfected clones carrying a marker for undifferentiated cells. Curr Biol 2001;11:514-518

12. Gerrard L, Zhao D, Clark AJ, Cui W. Stably transfected human embryonic stem cell clones express OCT4-specific green fluorescent protein and maintain self-renewal and pluripotency. Stem Cells 2005;23:124-133

13. Hohenstein KA, Pyle AD, Chern JY, Lock LF, Donovan PJ. Nucleofection mediates high-efficiency stable gene knockdown and transgene expression in human embryonic stem cells. Stem Cells 2008;26:1436-1443

14. Yao S, Sukonnik T, Kean T, Bharadwaj RR, Pasceri P, Ellis J. Retrovirus silencing, variegation, extinction, and memory are controlled by a dynamic interplay of multiple epigenetic modifications. Mol Ther 2004;10:27-36

15. Villa-Diaz LG, Garcia-Perez JL, Krebsbach PH. Enhanced transfection efficiency of human embryonic stem cells by the incorporation of DNA liposomes in extracellular matrix. Stem Cells Dev 2010;19:1949-1957

16. Chen G, Gulbranson DR, Hou Z, Bolin JM, Ruotti V, Probasco MD, Smuga-Otto K, Howden SE, Diol NR,
Propson NE, Wagner R, Lee GO, Antosiewicz-Bourget J, Teng JM, Thomson JA. Chemically defined conditions for human iPSC derivation and culture. Nat Methods 2011; 8:424-429

17. Mikkola M, Olsson C, Palgi J, Ustinov J, Palomaki T, Horelli-Kuitunen N, Knuutila S, Lundin K, Otonkoski T, Tuuri T. Distinct differentiation characteristics of individual human embryonic stem cell lines. BMC Dev Biol 2006;6:40

18. Spits C, Mateizel I, Geens M, Mertzanidou A, Staessen C, Vandeskelde Y, Van der Elst J, Liebaers I, Sermon K. Recurrent chromosomal abnormalities in human embryonic stem cells. Nat Biotechnol 2008;26:1361-1363

19. Beers J, Gulbranson DR, George N, Siniscalchi LI, Jones J, Thomson JA, Chen G. Passaging and colony expansion of human pluripotent stem cells by enzyme-free dissociation in chemically defined culture conditions. Nat Protoc 2012;7:2029-2040

20. Irion S, Luche H, Gadue P, Fehling HJ, Kennedy M, Keller G. Identification and targeting of the ROSA26 locus in human embryonic stem cells. Nat Biotechnol 2007;25:14771482

21. Badur MG, Zhang H, Metallo CM. Enzymatic passaging of human embryonic stem cells alters central carbon metabolism and glycan abundance. Biotechnol J 2015;10:1600-1611 

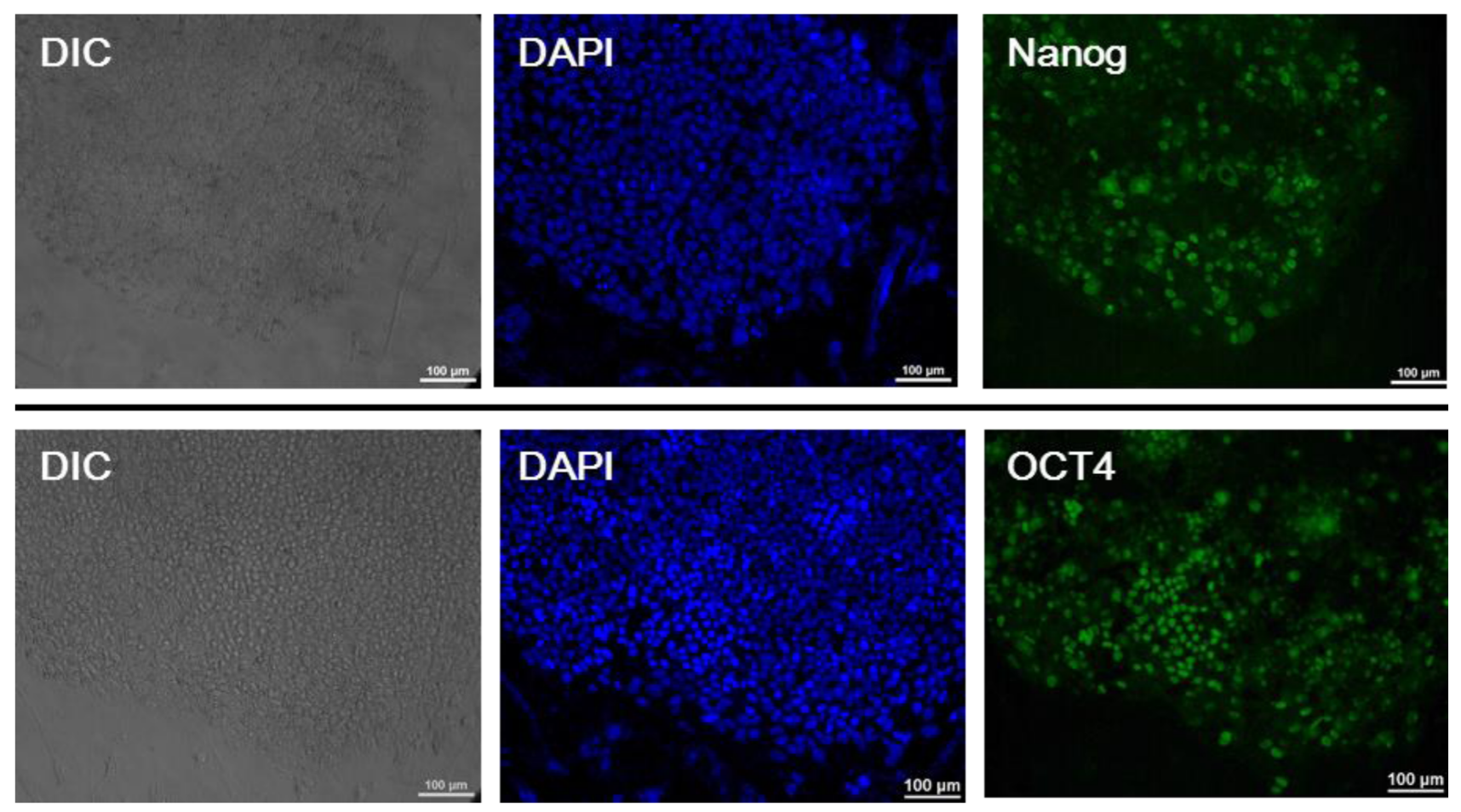

Fig. S1. Characterization of hESCs passaged by EDTA. Immunocytochemistry for stem cell-specific marker expression Nanog (green) and Oct4 (green); nuclei stained with DAPI (blue) and merged images. 


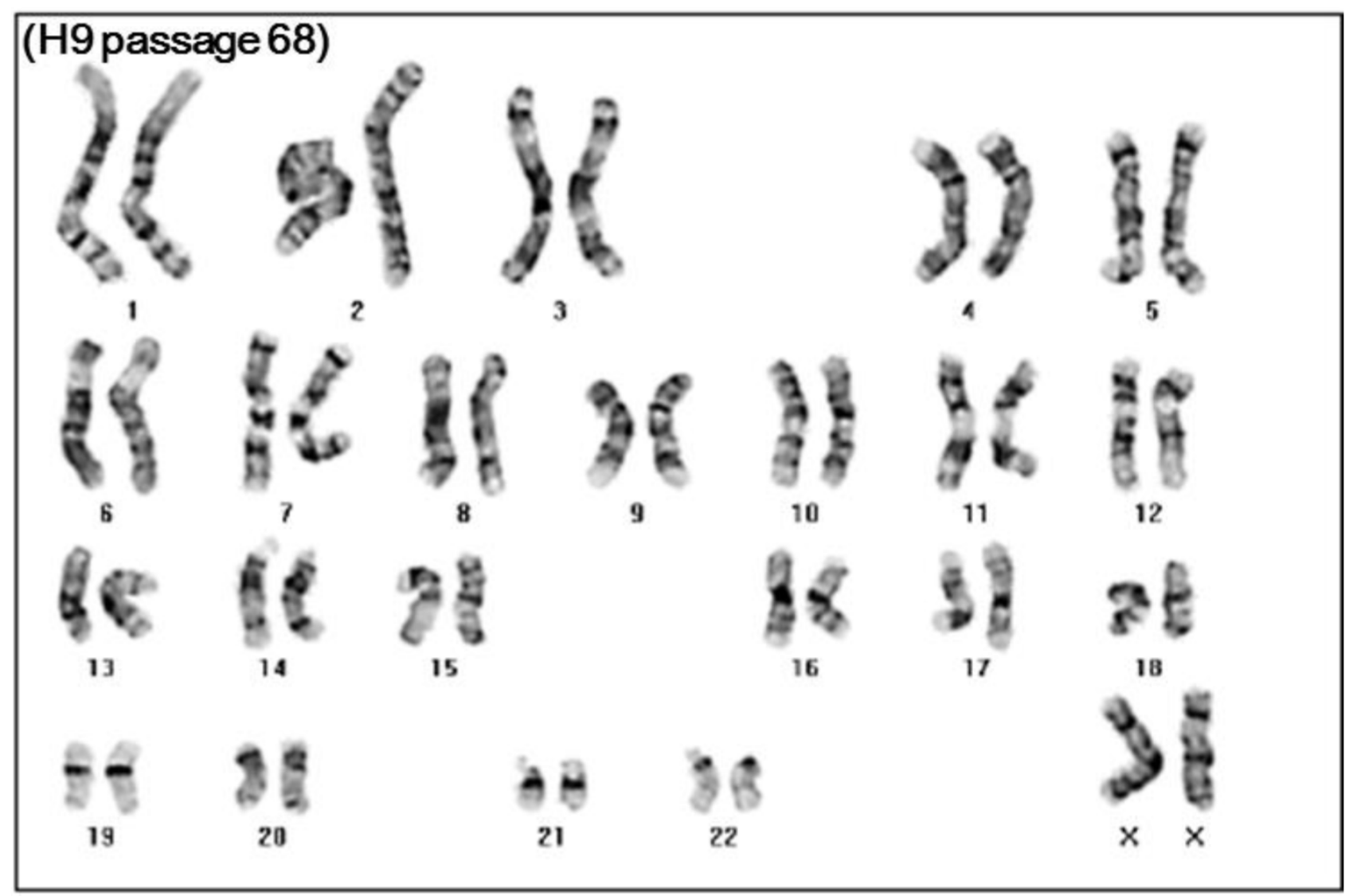

Fig. S2. Karyotype analysis of hESC. hES cell line (H9 passage 68) maintains a normal karyotype. A normal karyotype was observed for all analyzed nuclei. 

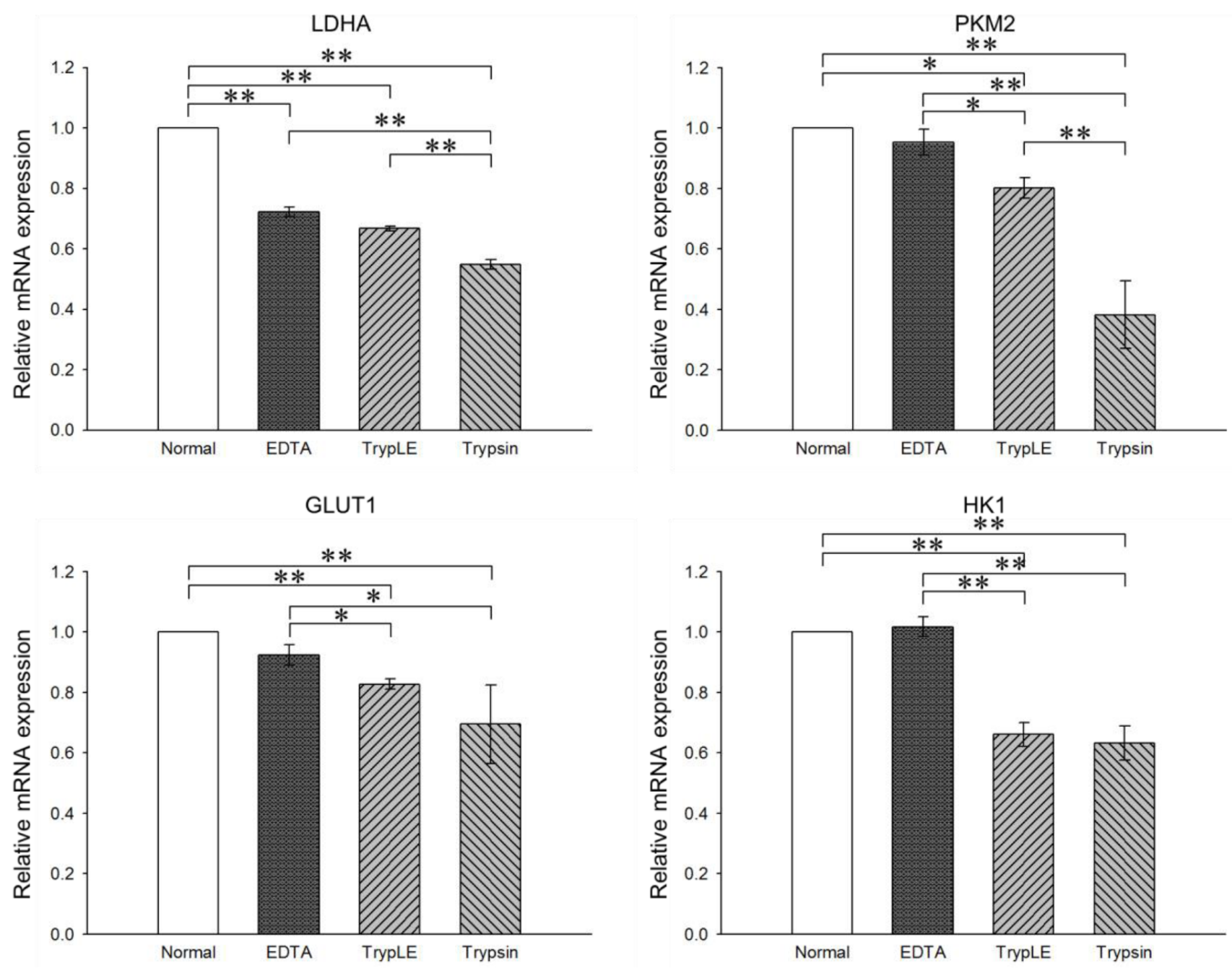

Fig. S3. The expression levels of mRNA involved in mitochondrial function at $2 \mathrm{hr}$ after seeding. Data represent the mean $\pm S D$. ${ }^{*} p<0.05$, $* * p<0.01$. 